Review

\title{
Vision and Control for UAVs: A Survey of General Methods and of Inexpensive Platforms for Infrastructure Inspection
}

\section{Koppány Máthé * and Lucian Buşoniu}

Automation Department, Technical University of Cluj-Napoca, Memorandumului Street no. 28, 400114 Cluj-Napoca, Romania; E-Mail: lucian@busoniu.net

* Author to whom correspondence should be addressed; E-Mail: koppany.mathe@ aut.utcluj.ro; Tel.: +40-264-401-587.

Academic Editor: Felipe Gonzalez Toro

Received: 27 December 2014 / Accepted: 26 May 2015 / Published: 25 June 2015

\begin{abstract}
Unmanned aerial vehicles (UAVs) have gained significant attention in recent years. Low-cost platforms using inexpensive sensor payloads have been shown to provide satisfactory flight and navigation capabilities. In this report, we survey vision and control methods that can be applied to low-cost UAVs, and we list some popular inexpensive platforms and application fields where they are useful. We also highlight the sensor suites used where this information is available. We overview, among others, feature detection and tracking, optical flow and visual servoing, low-level stabilization and high-level planning methods. We then list popular low-cost UAVs, selecting mainly quadrotors. We discuss applications, restricting our focus to the field of infrastructure inspection. Finally, as an example, we formulate two use-cases for railway inspection, a less explored application field, and illustrate the usage of the vision and control techniques reviewed by selecting appropriate ones to tackle these use-cases. To select vision methods, we run a thorough set of experimental evaluations.
\end{abstract}

Keywords: unmanned aerial vehicle; control; planning; camera-based sensing; infrastructure inspection

\section{Introduction}

Unmanned vehicles, including UAVs, offer new perspectives for transportation and services. Although the legal requirements are still quite restrictive [1], UAV applications are becoming 
widespread, from military usage to civil applications, such as aerial imaging [2] or various inspection tasks $[3,4]$.

We focus here on low-cost (under \$1500), small-scale (diameter under $1 \mathrm{~m}$ ) and lightweight (under $4 \mathrm{~kg}$ ) UAVs that can be reliably used outdoors. Examples for UAVs that fit these criteria are the Parrot AR.Drone [5], the Arducopter platforms [6] or others presented in Section 4. While the specific limits on size, weight and cost are, of course, arbitrary to an extent, we are also motivated to select them by the railway inspection application we discuss in Section 6: since the UAVs are small they are unlikely to damage a train in the event of an unavoidable collision, and their low cost makes them easily replaceable. More generally, inexpensive UAVs are accessible to civilian users and commercially attractive for companies, making them more likely to become widespread. Among small-scale UAVs, higher-cost platforms, such as the AscTec, Draganflyer and MikroKopter products, offer improved flight stability $[7,8]$ and advanced sensing units, such as laser rangefinders $[9,10]$ or thermal infrared cameras [11,12]. While such platforms are sometimes also referred to as low-cost UAVs [12,13], we consider here significantly less expensive UAVs.

UAVs under \$1500 use less expensive hardware, especially for sensing and processing units. They still possess basic navigation units, such as inertial measurement units (IMU) and possibly Global Positioning System (GPS) modules, but the measurement accuracy is usually reduced. Color cameras are used, which are useful only in daytime and do not provide depth and scale information for the captured environment. Nevertheless, the cameras are the richest data sources, so computer vision usually plays a central role in UAV automation. Building on vision, advanced sensing and control methods are used to compensate for the performance and capability limitations.

Therefore, in the first part of the paper, we survey general techniques for vision and control. We describe methods that work in any application, but are specifically motivated by infrastructure inspection, so we point out the connections of vision and control with this area. We begin by presenting at a high level existing vision methodologies and highlight those shown to be successful in UAV applications. The discussion is structured along several classes of techniques: feature detectors and descriptors to identify objects in the image, optical flow for motion detection and visual servoing and mapping techniques. The latter two techniques blur the line between vision and control, e.g., visual servoing tightly integrates visual feedback on the position relative to an object and the control actions taken to maintain the position. We continue by detailing UAV control methods, on two separate levels. For low-level stabilization and path following, we briefly introduce a simplified quadrotor model and methods for attitude and position control. We overview platform-independent high-level planning tasks and methods, focusing on methods that can integrate obstacle avoidance and other constraints.

Already for low-level control, and also for the remainder of our paper, we select quadrotors as our preferred class of UAVs. Among the two main types of UAVs, fixed-wing and rotorcrafts, rotorcrafts have the important capability of hovering. Subtypes are helicopters and multirotors, where multirotors are preferred for the robustness and modularity of the fuselage, being less subject to damage and easier to repair. Furthermore, quadrotors are the most widespread and least costly multirotors. Since we are interested in automating broadly-used UAVs, in this paper, we will focus on quadrotor platforms.

We start the second part of the paper by overviewing several low-cost quadrotor platforms. We then introduce the main focus of this second part, infrastructure inspection, and review UAV applications in 
this area. Selecting a less explored subfield, namely railway inspection, we develop two use-cases in this field to illustrate semi-autonomous short-range and fully-autonomous long-range inspection. We start with a detailed comparison of the performance of various feature detectors, based on real data from the use-cases. This also serves as a detailed illustration of the practical performance of many of the vision techniques we review. Based on the test results, we select appropriate vision algorithms. In addition, we select control methods based on our literature review, for each scenario, adapted to the facilities of the Parrot AR.Drone quadrotor.

In related work, a number of surveys address specific sensing, vision and control topics for UAVs. For example, the recent survey of Whitehead et al. [14] evaluates sensor types used for remote sensing applications. In the context of vision methods, [15] presents extensive datasets and benchmarks for optical flow techniques, and [16] discusses in detail edge detection methods. On the control side, [17] presents techniques for low-level stabilization and control, ranging from simple linear to more accurate nonlinear controllers, while [18] discusses high-level planning. Another survey [19] overviews planning methods from the perspective of uncertainties. The definitive book on robotic planning [20] also addresses low-level dynamics as constraints on planning. We do not intend to duplicate these efforts here. Instead, we provide an overall, high-level overview of both vision and control, focusing on methods relevant to recent low-cost quadrotors and infrastructure inspection applications. Indeed, we refer to these existing works, drawing on their comparisons between available methods and synthesizing their results, so in a sense, our paper is a meta-review of the area. Of course, due to the wide research fields discussed, the overview provided by us is not exhaustive. In particular, we do not include state estimation methods, used for example to infer unmeasurable variables from measured data or to perform sensor fusion. Our major goal is to help the practitioner in the areas of inspection with UAVs understand what methods are available and how they organize in a coherent landscape, so as to select an array of techniques for their specific application; and we provide the relevant references needed to implement the chosen techniques.

The next two sections present our survey on vision (Section 2) and control methods (Section 3). Then, Section 4 lists popular low-cost UAVs; Section 5 discusses common UAV monitoring and inspection applications; Section 6 evaluates vision techniques and selects control methods from those discussed for two illustrative use-cases; and Section 7 concludes our survey. Throughout, we pay special attention to sensor suites and present them whenever this information appears in the cited papers. Specifically, in Section 2.5, we discuss sensors used in vision-based applications. We highlight in Section 4 sensors found in low-cost platforms, and in Section 5.4, we present the sensor suites considered in infrastructure applications.

\section{Vision: Camera-Based Sensing and Image Processing}

Automated navigation of UAVs inherently requires sensing. Usually, ultrasonic sensors, color, thermal or infrared cameras or laser rangefinders are used to acquire information about the surrounding environment. From these sensor types, low-cost UAVs often possess color cameras. Information is then extracted using computer vision techniques: the acquired images are processed for stabilization, navigation and further information collection. 
In UAV navigation, feature detectors and extractors are often used for object detection; optical flow techniques are used to distinguish motion in a scene; visual servoing is employed to translate image frame motion into UAV displacement; whereas 3D reconstruction methods are exploited for navigation and mapping. The literature on computer vision is rich (see surveys [15,21-23]), and instead of reproducing these efforts, here, we briefly introduce the aforementioned classes of vision techniques, highlighting popular methods and providing a list of relevant references for further reading. We also exemplify the use of specific methods in inspection applications. Later on, in Section 6, we will evaluate the performance of existing implementations of several vision methods on real data taken from our railway inspection use-cases.

An important remark regarding most vision methods is that they are well known as being difficult to rank by performance. Each method is suitable for specific types of environments and target objects. Evaluation methodologies, like the one presented by Rockett [24], exist, but the performance of vision methods remains subject to the fine-tuning of their parameters according to the problem at hand. Nevertheless, certain methods are preferred either for their robustness, flexibility in parameter selection or lower computational demands, as highlighted in the sequel.

\subsection{Feature Detection and Description Methods}

Feature detection and description algorithms are basic tools for object detection and tracking. These methods are used, for example, to extract UAV position and motion information. Methods differ from each other in the preprocessing used (grayscaling, blurring, masking), in the way the features are interpreted and selected, and in the mathematical operations used in the processing steps.

Features determine regions of interest in images, which are classified roughly as edges, corners and blobs. Detection methods are responsible for identifying them, whereas descriptors are used to match a feature in two images (e.g., images from a different perspective or subsequent frames from a video stream). Detectors in combination with descriptors and matching methods form complete tools for motion tracking. They can be also used for object detection given a reference image of an object, and in this context, additional tools, like the model fitting methods, can be considered. In what follows, we first discuss edge, corner and region detectors, then descriptor methods. We present then feature matching, homography-based detection and model fitting methods for object detection.

Edge detection is usually employed to identify lines and planes in images. Some of the classic methods are Canny, Sobel, Laplacian and Scharr edge detectors [25]. Several surveys exist that compare the performances of these and other algorithms [21,26,27]. A survey performed by Oskoei et al. [16] highlights the good performance of step edge models used for feature lookup and Gaussian filtering considered for further image processing. A classic example of such a method is the Canny edge detector. However, it is known to produce false edges for noisy images, and therefore, methods like the Haar wavelet transform [26] can be considered when the performance of the former is not satisfactory.

Corner and region detectors are mainly used for finding and tracking objects. The Harris-Stephens [28] and Shi-Tomasi [29] methods are often used. A recent study performed by Tulpan et al. [30] compares four corner detectors in their performance of identifying distant objects for sense-and-avoid applications. They compare the Harris-Stephens, smallest uni-value segment 
assimilating nucleus (SUSAN) [31], features from accelerated segment test (FAST) [32] and Shi-Tomasi methods on real video streams. Their results show that the Shi-Tomasi and Harris-Stephens methods outperform the others when looking at the execution time, while the Shi-Tomasi method has the best results concerning the detection range and the ratio of frames containing the detected target.

Feature descriptors are used for matching features in image pairs, either for detecting motion or for finding objects. Well-known methods are speeded up robust features (SURF) [33] and scale-invariant feature transform (SIFT) [34], whereas from recent years, we mention binary robust independent elementary (BRIEF) [35] and oriented FAST and rotated BRIEF (ORB) [36]. SIFT and SURF are older methods and are superseded by BRIEF and ORB in execution time while keeping comparable accuracy. On the other hand, ORB overcomes the limitations of BRIEF in processing rotations in images.

Given the feature descriptors, features can be matched in pairs of images. This can be achieved by simply comparing the descriptors from the two processed images and marking the closest descriptor pairs. This approach is called brute force feature matching. Other solutions consider search trees for comparing the descriptors. A popular matcher uses approximate nearest-neighbor (ANN) search [37] that offers a more efficient way to match features than the brute force approach. A well-known implementation of ANN is the Fast Library for ANN (FLANN) [38]. Using matched features, methods like the homography transform [39] determine the transformation of the image compared to the reference image, and from there, they infer displacement and rotation. A recent comparison of camera pose estimation algorithms using stereo cameras shows that the homography method provides an acceptable level of detection and is useful for applications with computational constraints [40].

Model fitting methods are often used in object detection. They categorize image features to find inliers and outliers according to a model (a curve or a shape described mathematically). A well-known example is the random sample consensus (RANSAC) method. A comprehensive performance evaluation of the RANSAC family is performed by Choi et al. [23], showing an accuracy and robustness improvement for maximum likelihood estimation SAC (MLESAC). RANSAC is used in several UAV applications, e.g., for wall plane detection [41] or for identifying the ground plane [42,43]. Although RANSAC methods aid the identification of objects having various shapes, they require high processing power and, thus, are less preferred in applications where computation is limited. Another classical model fitting method class uses the Hough transform; see surveys [44,45]. Hough transforms are most often used as straight line detectors and, thus, are preferred for linear structure detection. From the numerous variants [45], we point out the progressive probabilistic Hough transform for its faster computational performance and for the available implementation in OpenCV [46].

In UAV inspection applications, feature detectors are useful for detecting targets (buildings, objects) or references that have to be followed (like linear structures or the horizon). For linear structure detection, edge detectors can be combined, e.g., with line extractors. To achieve target detection, feature detectors can be coupled with descriptors, matchers and reference images. When combined with descriptors and matchers, feature detectors can also be used to track moving objects or to keep a reference position relative to a detected object. 


\subsection{Optical Flow Techniques}

Optical flow is a family of techniques that focuses on determining motion from images. More precisely, optical flow can be defined as the apparent motion of feature points or patterns in a 2D image of the 3D environment [47]. Often, optical flow detection is performed, e.g., with an optical mouse sensor, as it works on a similar principle and is a popular, well-tested device [47]. A comprehensive analysis of existing optical flow techniques is performed by Baker et al. in [15]. As stated in their concluding remarks, at the time of their publication, the most promising optical flow detection approach was the one presented by Sun et al. [48]. In a more recent study by Sun et al. [49], they show, among others, the good performance of classic optical flow formulations.

Although providing useful navigation information, optical flow algorithms are usually time consuming. Chao et al. [47] remark for instance that usually, optical flow algorithms perform well with image sequences presenting much slower motions compared to UAV flights. These methods can nevertheless still be considered, for example, in near-hovering operation of UAVs.

\subsection{Visual Servoing}

Building on the techniques discussed before, visual servoing deals with controlling the camera (and, therefore, vehicle) motion using visual data. In the context of UAV navigation, visual servoing methods offer solutions for translating image frame motions into real-world displacement. Examples of applications of visual servoing in inspection are flight around traffic signals, following gas pipeline or scanning building façades.

In its classical formulation, visual servoing builds upon feature detectors. Novel approaches consider other parameters (mainly global histogram parameters) of the images as features and perform visual servoing using this information [50-52]. We concentrate next on feature-based visual servoing, which has two major directions: position-based visual servoing (PBVS) and image-based visual servoing (IBVS). PBVS finds a position estimate of the camera by calculating the pose of a known object in the image and uses this position to correct the motion path [53]. IBVS simply selects and tracks features in the images, using feature descriptors shown in Section 2.1, and corrects the position of the camera so as to keep the desired position of the features in the image frame [54]. Although simpler, IBVS may suffer from drift, and thus, it is often used only in combination with other navigation methods (e.g., GPS/IMU-based control) or as a fallback solution. The pros and cons are thus complementary for the two approaches: PBVS can ensure accurate positioning, but at high computational costs, while IBVS is faster, but may result in following undesired trajectories. Implementation examples and further discussions can be found, e.g., in papers $[55,56]$.

\subsection{D Reconstruction Methods: Mapping}

Beyond finding the pose and the motion of the camera, simultaneous localization and mapping (SLAM) constructs a map of the environment while localizing the vehicle on this map. Localization then allows for autonomous navigation in unknown environments. Of course, SLAM is useful for mapping applications. In the case of camera-based SLAM, depth information, needed for finding distances and 
scaling factors of objects, can be collected by means of extra sensors or by using reference tags. For low-cost solutions, the latter technique is preferred, which calculates the distance from the size of known objects or tags, captured by the camera.

SLAM requires high processing performance, and its implementation is time consuming. A common implementation of SLAM is parallel tracking and mapping (PTAM). PTAM addresses the processing issue by executing UAV tracking (localization) and mapping in two parallel threads: the first thread tracks the camera motion (localization step), while the second one adds new information to the map (map refinement step) [42]. Despite parallel execution, PTAM remains a computationally-consuming algorithm, especially due to the map that requires more processing as it grows.

Yang et al. [42] consider PTAM to find an indoor landing site autonomously, using a single down-looking camera. They propose to obtain a constant computation time of PTAM by avoiding refinement of the entire map and performing it only locally, around the current position of the quadrotor. Similarly, Schauwecker et al. [43] consider refining maps only locally and clearing old frames in order to boost PTAM. They find that using down-looking cameras, the algorithm can have problems in tracking the quadrotor's motion in the case of yaw rotation (around the $z$ axis). Using stereo cameras, they can correct for these errors in the case of slow rotations.

Although research in the field of computer vision is extensive, there remain several open challenges. Most of the vision algorithms are subject to fine-tuning and are limited in use to certain conditions, specifically due to illumination conditions, pattern types and the motion of captured objects. Another issue is the increased processing time of these methods, which needs to be further reduced for online applications on devices with limited processing power. Additionally, the lack of scaling and distance information in the case of 2D images leads to the need for further tools (sensors or techniques) for acquiring this information. Though the existing solutions are promising, further development is needed for having more robust techniques with wider applicability.

\subsection{Sensors in Image Processing Applications}

From the above listed works, several provide details on sensor platforms. We have found, e.g., that Tulpan et al. [30] use a Prosilica GC2450 5 MP monochrome camera for image processing, operating at $14 \mathrm{fps}$. Flores et al. [41] consider Kinect, a commercially available camera system, consisting of a low-cost RGB-D and infrared camera, providing $640 \times 480$ pixel RGB images and $320 \times 240$ pixel depth images, both at $30 \mathrm{fps}$. Yang et al. [42] work with a Firefly MV monochrome camera that provides $640 \times 480$ pixel images at $60 \mathrm{fps}$ and a 90-degree viewing angle. Schauwecker et al. [43] consider a dual stereo-camera solution, mounting two pairs of $640 \times 480$ grayscale cameras on their UAV, one pair facing downwards, recording at $15 \mathrm{fps}$, and another facing ahead, recording at $30 \mathrm{fps}$.

One may conclude that, in most cases, images not larger than $0.3 \mathrm{MP}$ are used for image processing, at frame rates up to $30 \mathrm{fps}$. This resolution is far lower than those offered by the currently available cameras, though it is preferred for keeping computation low (higher resolution images would require more processing time) and also for requiring only low-cost devices. Most research results, including the above ones, show that this resolution is good enough for proper detection. Furthermore, in terms of 
control, the acquisition frame rate of $30 \mathrm{fps}$ is high enough, e.g., for controlling small-scale UAVs, and exceeds, in many cases, the processing rate that vision methods can offer.

\section{Flight Control and Planning}

Relying on information provided by sensing, effective control can help in overcoming the limitations of inexpensive sensors. Indeed, several works report good results using low-cost platforms with advanced controllers [57-59]. In general, the task of an unmanned aircraft is to safely navigate on a desired path and/or visit points of interest in order to perform certain missions. Control tasks behind these terms were grouped by Amelink [60] in the following levels of abstraction, from top to bottom: mission, navigation, aviation, flight control and flight. In this manner, he clearly classifies control problems and offers a modular approach to UAV control.

Instead of such a detailed decomposition of UAV control problems, we prefer to discuss control tasks at two levels: low-level flight control and high-level flight planning. With this grouping, the lower level covers control tasks that are often already implemented in UAVs: hovering ability and disturbance rejection, achieved mainly by attitude control, and trajectory following, the result of position control. By an abuse of terminology, we will refer to all of these tasks as UAV control in this section. On the other hand, high-level flight planning is then made responsible for mission and path planning, including obstacle avoidance. We will call these problems together UAV planning.

Many of the methods presented, especially the higher level planning techniques, are platform independent. Low-level control will be discussed from the perspective of quadrotors, the platform type we will use in our illustrative use-cases and also in our future work. Therefore, first, we introduce the dynamic model of a quadrotor, followed by detailing low-level control and high-level planning methods.

\subsection{Quadrotor Dynamics}

A basic scheme of a quadrotor is shown in Figure 1, where $\mathscr{E}$ denotes the Earth frame, also called the inertial frame; $\mathscr{B}$ denotes the body frame, attached to the center of mass of the quadrotor; $x, y$ and $z$ mark the coordinates of the center of mass of the quadrotor, in the Earth frame $\mathscr{E} ; \phi, \theta$ and $\psi$ correspond to the conventional roll, pitch and yaw angles; and $\omega_{i}$ marks the angular velocity of each rotor separately.

In the "plus" configuration, where the vehicle axes correspond to the $x$ and $y$ axes of frame $\mathscr{B}$, displacement along the $x$ axis can be obtained by pitch rotation, which results from keeping $\omega_{1}=\omega_{3}$ and setting $\omega_{2} \neq \omega_{4}$. Similarly, flight on the $y$ axis results from yaw rotation, i.e., $\omega_{2}=\omega_{4}$ and $\omega_{1} \neq \omega_{3}$. Hovering, lift and landing can be achieved by having velocities of the same magnitude on all propellers, whereas rotation around the $z$ axis is the result of $\omega_{2}=\omega_{4} \neq \omega_{1}=\omega_{3}$. Then, control commands can be defined as:

$$
\begin{aligned}
& U_{\text {coll }}=b\left(\omega_{1}^{2}+\omega_{2}^{2}+\omega_{3}^{2}+\omega_{4}^{2}\right) \\
& U_{\phi}=b\left(\omega_{1}^{2}-\omega_{3}^{2}\right) \\
& U_{\theta}=b\left(\omega_{4}^{2}-\omega_{2}^{2}\right) \\
& U_{\psi}=d\left(\omega_{2}^{2}+\omega_{4}^{2}-\omega_{1}^{2}-\omega_{3}^{2}\right)
\end{aligned}
$$

where $U_{\text {coll }}$ denotes the collective input (responsible for vertical displacement), $U_{\phi}$ the roll ( $y$ axis movement), $U_{\theta}$ the pitch ( $x$ axis displacement) and $U_{\psi}$ the yaw forces, $b$ is the thrust coefficient and 
$d$ is the drag coefficient. With these four inputs, the quadrotor can be operated simply in non-acrobatic flight mode (non-acrobatic flight maneuvers mean that the quadrotor's velocity is changed slowly and the vehicle is used to fly most of the time parallel to the Earth, up to some tilt being necessary for horizontal displacement). Note that all of these motions are with respect to frame $\mathscr{B}$, which then have to be transformed into Earth frame $\mathscr{E}$.

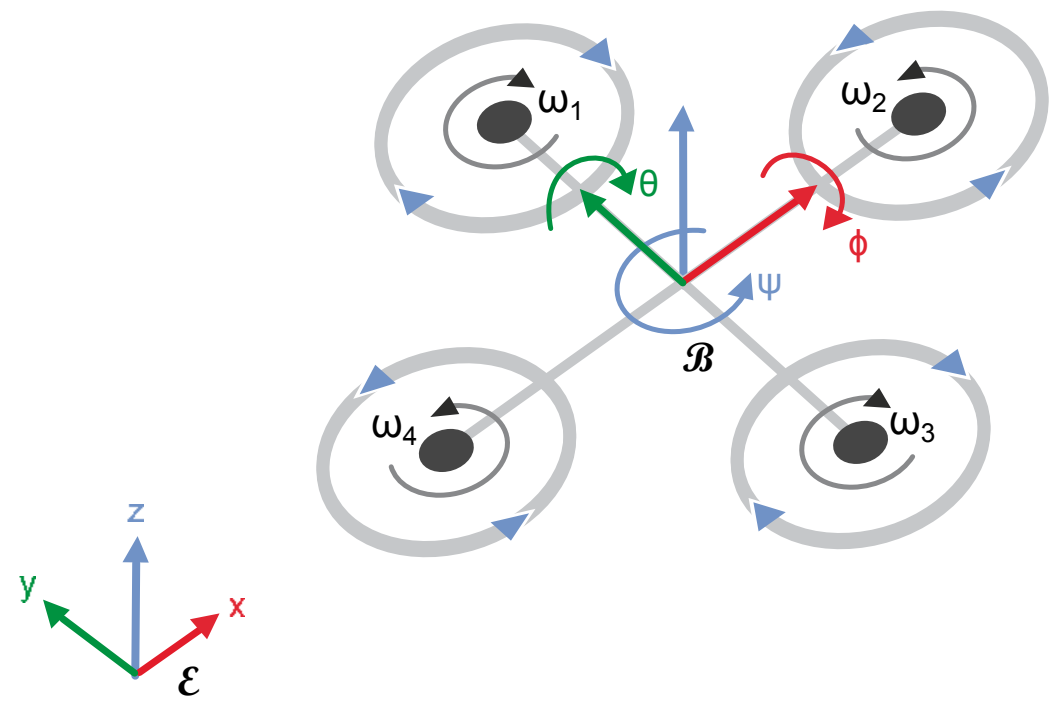

Figure 1. Quadrotor model.

Now, the reaction to the control inputs, i.e., the flight dynamics of the quadrotor, can be modeled using the Euler-Lagrange approach. A simplified form of the dynamics in near-hovering mode can be written as [8]:

$$
\begin{array}{cll}
\ddot{x}=\theta g & \ddot{y}=-\phi g & \ddot{z}=\frac{\Delta U_{\text {coll }}}{m} \\
\ddot{\phi}=\frac{l}{I_{x}} U_{\phi} & \ddot{\theta}=\frac{l}{I_{y}} U_{\theta} & \ddot{\psi}=\frac{1}{I_{z}} U_{\psi}
\end{array}
$$

Often, more general forms of this model are considered [61,62]. However, even those works build on the assumptions of near-hovering operation mode, low flight speeds and that the quadrotor can be modeled as a rigid body and has a symmetric structure. As these are realistic considerations for most low-cost small-sized quadrotors, controllers based on the principle of Equation (2) can be easily transferred from one platform to another, where only some parameters have to be adjusted to match the new vehicle.

\subsection{Low-Level Flight Control}

The main low-level control tasks are: achieving flight, stabilizing the UAV and following a flight path. These tasks are addressed by attitude and position control, which, in the case of quadrotors, are commonly coupled as a nested control loop, shown in Figure 2. Attitude control is then responsible for flight stabilization and tracking the desired heading, while position control serves for trajectory following. 


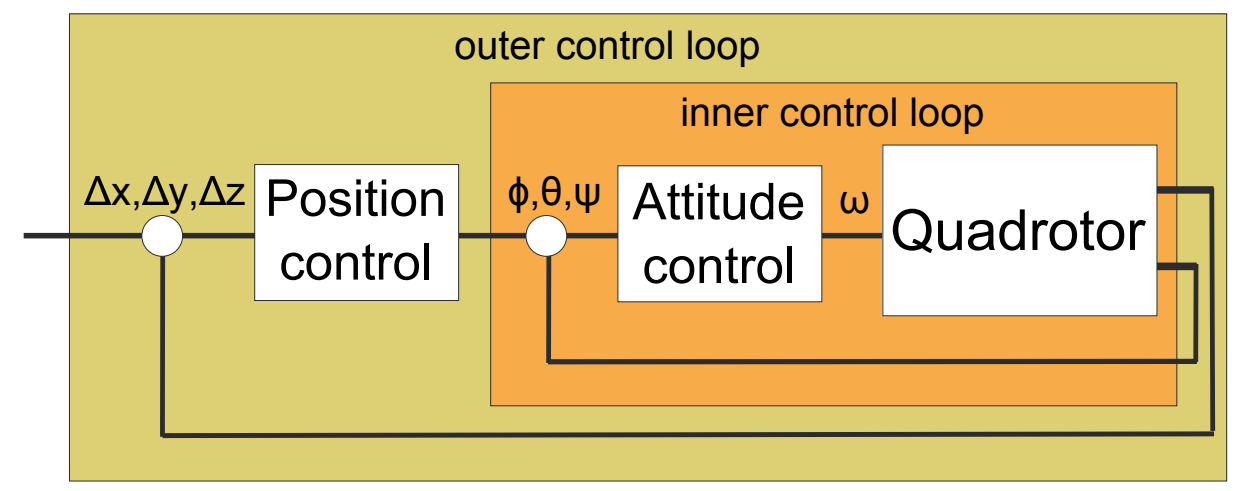

Figure 2. Nested low-level control loop: attitude and position control.

Attitude control is often addressed by using proportional integral derivative (PID) controllers. Often, the PID controllers are set up by experimental tuning. They have the advantage of requiring no complex model of the system dynamics. Based on the results from papers [57,63], this control method, although simple, provides good results for the attitude control. However, the attitude controller is usually enhanced with robust features for obtaining improved stability [57,64].

Concerning position control, Raffo et al. [64], for example, propose to use an error-model based model-predictive control that simulates a virtual quadrotor following a desired trajectory. The role of their controller is to minimize the position error between a real and a virtual quadrotor. They compare this solution in a simulation to a backstepping approach. Both methods show robust tracking performance, though the former solution leads to smaller errors and smoother control.

Attitude and position control are often discussed together. Cui et al. [63] perform, for instance, trajectory tracking, using PID controllers for position control and several controller types for setting the attitude of the simulated vehicle. In simulations, they show that the PID controller provides the smallest tracking error and lowest settling time for attitude control. Salazar et al. [62] combine PID with robust sliding mode control (SMC) for attitude and position control in performing trajectory tracking. They conclude that, despite the good control performance of SMC, chattering of the control input may lead to quick wearing of the actuators, and thus, the use of SMC might be a less preferred solution.

Despite the good performance of current low-level stabilization systems and controllers, several further challenges remain to be addressed. Among these are the various types of uncertainties appearing in outdoor operation, the integration of saturation limits in the control schemes and the underactuated nature of the systems. In recent works, such challenges were addressed by more advanced control methods, such as adaptive controllers [8] or model-predictive control [65].

\subsection{High-Level Flight Planning}

The higher level problems of UAV automation relate mainly to defining and planning missions, as well as to planning flight paths that fulfil these missions. The goal is to make UAVs fly autonomously based on a mission plan and to make the flight paths feasible and optimal.

Path planning provides flight paths for the lower-level trajectory tracker. It results in optimization problems where certain costs (e.g., energy consumption, execution time) have to be minimized to find an optimal path. To achieve online execution, often, receding horizon techniques are considered. In the 
sequel, we detail the principle of receding horizon planning and present works that use it for UAV path planning. Afterwards, we discuss planning constraints that ensure flight path feasibility and methods to address them.

Exhaustive overviews of path planning methods can be found in [18-20,66]; see also the references therein. Here, we provide some reference works in the field of UAV path planning.

The idea of receding horizon planning is to reevaluate solutions while closing the control loop. In control, this approach is called model predictive control (MPC). At each call, MPC evaluates possible control sequences for a period of time called a horizon. Each sequence has an associated cost. After the simulation stops, MPC follows the control sequence with the best cost for a time period called the execution horizon. The algorithm is then repeated from the new state. MPC can cover nonlinear dynamics problems, too, such as UAV path planning and path following under uncertainties. However, especially in that case, MPC becomes time consuming, mainly due to "repeated" calculation of the cost values that usually requires simulating the model of the system.

For reducing computation, a common planning method used for UAV planning is the rapidly-exploring random tree (RRT) algorithm. RRT propagates random samples in the search space of control sequences, rapidly covering in this manner the possible solutions. Lin et al. [66] use RRT in a receding horizon fashion and couple it with Dubins curves in order to find feasible paths online, paths that avoid moving obstacles. Dubins curves are curves that connect two points while respecting constraints on the curvature. Although the RRT method cannot ensure global optimality [66], shows the practical success of the method both in simulations and in real flights.

Bellingham et al. [67] use MPC for path planning among obstacles. They work with linearized models and simplify the cost value calculation by using a cost estimator in order to reduce the execution time. They manage to obtain near-optimal solutions in around a minute [67]. More recent works report on using MPC for more complex situations, such as path planning for multi-UAV formation flights among obstacles [68] and with communication constraints [69]. However, the computational demand of these solutions is not transparent and is likely to exceed the processing performance of low-cost platforms.

An important challenge comes from the feasibility constraints considered in the planning. These integrate kinematic constraints (coming, e.g., from obstacles) and dynamic limitations (mainly due to the velocity and acceleration limits of the vehicle) [70]. Feasibility constraints can be treated in several ways. Commonly, they can be implemented as equality or inequality constraints in the optimizer $[67,68,71]$ or can be simulated as curves [66,72,73]. For example, mixed-integer linear programming (MILP) is an optimizer that can integrate both linear and binary constraints. Bellingham et al. [67] use, for instance, MILP for solving path planning, while considering both the continuous constraints resulting from the dynamics of the vehicle and binary constraints coming from obstacle avoidance rules. In their RRT approach discussed before, Lin et al. [66] use Dubins curves for their planning method in order to cover the dynamic constraints of the vehicle.

Alternatively, kinematic constraints (e.g., obstacles) in particular can be addressed by means of control methods from computer vision; see Section 2. Navigation methods, such as visual servoing or control based on optical-flow, can be considered. Furthermore, to reduce the problem complexity, obstacle avoidance can be addressed with position control, where the avoidance maneuver represents a temporary deviation from the planned trajectory [74]. Similarly, dynamic constraints can be applied to 
adjust the flight path after it has been planned. Methods, such as the artificial potential field, can be used to smoothen the trajectory [75]. Such solutions are often less time consuming and may be preferred when computation power is limited. However, they do not offer performance guarantees and might be applicable only in certain scenarios, where waypoints are not mandatory to be reached, but deviations from the planned trajectory that are small in a certain sense are acceptable.

On top of flight planning sits mission planning, which covers the descriptive part of UAV automation. Mission planning includes all formal and informal requirements and specifications regarding usage of the vehicle. Furthermore, it is responsible for translating the missions into a well-defined sequence of subtasks that can be interpreted by path planners. Such subtasks are: take-off, fly to a coordinate, track an object, etc. Mission planning can be formalized, and the translation of missions into subtasks can be then performed automatically, based on a set of subtasks and rules. Looking at the literature, only sparse work has been done in this direction, mainly related to airspace integration efforts $[1,76,77]$.

Several open challenges exist in UAV high-level planning. The computational demand of the advanced path planning methods is often high compared to the processing capabilities of low-cost UAVs, meaning that adaptations of these techniques are required in order to implement them onboard and online. Computational limitations also require approximate models, linearization or discretization of the system. Regarding the automation tasks, obstacle avoidance remains the most demanding problem, mainly due to the big variety of avoidance cases, which are hard to address all at once. On the other hand, mission planning challenges mainly relate to airspace integration issues, for which it is important to have a proper formalization of tasks in order to clearly define the expected and possible flight phases and events.

\subsection{Flight Control and Planning Methods for Inspection Applications}

Low-level stabilization is a basic flight requirement, and thus, attitude and position control are mandatory. However, the required flight planning techniques will vary. If one can assume obstacle-free flight when keeping a reference distance to the inspected target, visual servoing or preplanned trajectories can be considered. For online obstacle avoidance or for power consumption optimization online replanning, methods like the RRT should be considered. MPC-based techniques are able to explicitly deal with more complex constraints on the inspection (e.g., covering both flight time and obstacle avoidance) and are thus preferred in environments with more obstacles (e.g., urban roads, train stations).

\section{Low-Cost Quadrotor Platforms}

Having overviewed vision and control techniques, we move on to available low-cost platforms and UAV applications. The market of low-cost UAVs has exploded in the past two years. New brands and models of UAVs continuously appear, mainly quadrotors and helicopters, and several communities [78,79] and websites [80-82] focus on the evolution of these products. Instead of looking at the newest platforms resulting from startups and other research projects, we focus on popular brands. Working with a widespread platform usually ensures that the hardware is well tested and that the platform has long-term support, compared to more custom solutions. We therefore list several representative UAV platforms, available until April 2015, that also satisfy our criteria defined in Section 1. 
We focus mainly on ready-to-fly (RTF) vehicles, having the advantage of less time needed for setup and calibration. However, RTF vehicles are usually limited in programmability and, therefore, are less customizable. This aspect does not limit the manually-teleoperated inspection applications, but is relevant for automated flights. Thus, we discuss with each presented platform the level of customization, as well. We also compare these platforms with a category of modular solutions that overcome these limitations. For each platform, we highlight the sensors used. In general, all of the quadrotors listed have onboard stabilization, some type of camera (embedded or as a separate device) and wireless connection for data transmission, and most of them include a GPS module, as well. Details are presented below.

Parrot released its popular AR.Drone 2.0 in 2012 citeweb:parrot. Edition 2.0 costs $\$ 400$ and weighs $420 \mathrm{~g}$. It has two onboard, built-in cameras, a bottom quarter video graphics array (QVGA) camera $(320 \times 240$ px resolution) with 60 fps for ground speed measurement and an HD front camera $(1280 \times 720$ px resolution) with $30 \mathrm{fps}$ for video recording. The flight stabilization system consists of a set of three-axis gyroscope, accelerometer and magnetometer, enhanced with a pressure sensor that, together with the previous, makes the quadrotor able to withstand winds of up to $24 \mathrm{~km} / \mathrm{h} \mathrm{[83].} \mathrm{A}$ pair of ultrasonic sensors help in altitude keeping in close-to-terrain flights (up to $6 \mathrm{~m}$ ). The onboard flight controller runs in Linux and is closed-source; however, using the Parrot SDK or other APIs, one may remotely access functionalities, such as: receive online camera image frames, navigation and other sensor data and issue high-level velocity commands on all axes, as well as take-off and land commands. These operations are limited to the range of the WiFi signal used for communication with the quadrotor. Various projects target extending the control range or allow for onboard programmability, though not as part of the official product. An official extension by Parrot is a GPS module that, for an additional $\$ 120$, among others, allows for flight path scheduling by specifying waypoints, even outside the WiFi range. However, the quadrotor is not controllable outside the WiFi range, which limits it to short-range usage. Besides this popular model, Parrot released the Bebop drone at the end of 2014. For \$500, among others, Bebop provides improved processing image capturing capabilities (14 Mpx image and $30 \mathrm{fps}$ video recording at $1920 \times 1080 \mathrm{px}$ ), has a built-in geo-location system (GNSS, including GPS and GLONASS) and an operation range of $250 \mathrm{~m}$ [84]. The additional Skycontroller, costing around $\$ 400$, allows for extending the operation range to $2 \mathrm{~km}$.

Similar products are the Xaircraft X650 Pro [85], having a SuperX onboard flight controller [86], and the Mikrokopter Quadrokopter XL [87], using the FlightCtrl ME flight controller [88]. The price of these UAVs is around $\$ 1000$, and they weigh around $1000-1500 \mathrm{~g}$. Based on the flight controller specifications, both controllers use for stabilization a set of sensors like those presented with the AR.Drone: pressure sensors, three-axis gyroscopes, magnetometers and accelerometers. A special feature of the controller of the Mikrokopter UAV is that its altitude sensing works up to $5000 \mathrm{~m}$. Both UAVs are meant for mounting external cameras, where the X650 Pro flight controller has built-in functionalities for the camera gimbal stabilization and control. Theoretically, any recording device can be attached to these UAVs, up to the payload limit (around $1 \mathrm{~kg}$ for both UAVs).

DJI produces quadrotors for aerial imaging [2]. For the price of around $\$ 1000$ and weights of about $1200 \mathrm{~g}$, these vehicles are known to be stable. However, the flight controllers used with them allow only for path scheduling based on GPS waypoints, using a graphical interface. The publicly available specifications provide less technical information about the flight controllers [89]. Some of 
their proprietary flight controllers support the attachment of a GPS module. The newer platforms have proprietary cameras attached to the UAV through a two-axis gimbal, providing HD $1080 \mathrm{p}$ recording at $30 \mathrm{fps}$, and taking $14 \mathrm{MP}(4384 \times 3288 \mathrm{px})$ photos. However, certain platforms support the use of other cameras than the proprietary ones. Furthermore, DJI specifies an extended range of operation for the newer products, up to $2 \mathrm{~km}$.

In contrast with the above-listed RTF vehicles, which are readily assembled UAVs with the possibility of limited customization, 3D Robotics and the Arducopter come with modular solutions, based on the Arduino platform [90]. These UAVs use Ardupilot Mega (APM) or Pixhawk flight controllers that are known to be custom-programmable. Furthermore, these controllers support the attachment of various external devices, such as sensors, controllers and communication units. The Arducopter UAVs are usually custom-built, but there exist complete kits, as well, offering RTF or almost-RTF solutions. An RTF quadrotor is the 3DR Iris+, which costs $\$ 750$ and weighs $1300 \mathrm{~g}$ [91], whereas an almost-RTF solution is the Arducopter Quad [6], costing \$620 and having a similar weight. Compared to the platforms from the other producers, the Arducopter UAVs are highly customizable, though requiring more knowledge of UAV programming and operation. A more recent platform is the 3DR Solo, a user-friendly RTF UAV [92] with enhanced capabilities (among others, increased flight time up to $20 \mathrm{~min}$ with a mounted GoPro camera and an improved onboard controller).

Regarding sensors in Arducopters, these platforms come with the ability of customization. The newer Pixhawk controller comes with built-in gyroscopes, accelerometers and pressure sensors [93]. On the vision part, the Iris+ has, for instance, the possibility of mounting an external camera using a gimbal system. Pixhawk offers an optical flow module called PX4FLOW [94], which can be used for navigation purposes. It is not meant for video recording, though, due to its reduced performance (it has a resolution of $752 \times 480 \mathrm{px})$.

The presented UAVs are meant mainly for aerial imaging and gaming applications. Despite the different price ranges, the types of sensors used for stabilization are similar. Obviously, the more costly solutions offer better stabilization. On the vision side, platforms supporting the mount of external cameras offer improved recording experience. However, working with integrated vision units eases the usage of the platform, as in the case of the AR.Drone or with the PX4FLOW module. The quality and frame rate offered, for example, by the AR.Drone 2.0 are already good enough for use for vision-based navigation and environment capturing.

\section{UAVs for Infrastructure Inspection Applications}

A growing interest is shown for using UAVs for inspection and other remote sensing applications, starting from public area monitoring, to infrastructure inspection, intelligent farming or aerial mapping. Reaching satisfactory performance with low-cost UAVs can offer new perspectives for industrial applications and public services.

As we discuss in the sequel, several projects already focus on inspection use-cases, although they use more costly UAVs. We present specific applications by grouping them into two common classes, namely power line inspection and building monitoring. We also dedicate a subsection to railway inspection 
applications, a less explored field. Furthermore, we highlight the level of autonomy of the UAVs used in the works discussed. Finally, we list and discuss briefly the sensors used in the presented applications.

\subsection{Power Line Inspection}

Power line inspection applications roughly cover the tasks of following the lines and stopping at interesting points, to scan in detail certain parts of the infrastructure. This procedure is similar to the case of inspecting pipelines or any other linear structures, such as roads, walls, coasts, etc. Although projects can be found that target gas pipeline monitoring [95] or structure inspection in general [96], the topic of power line inspection appears to be more popular in recent years.

In their survey in 2010, Montambault et al. [97] already point out several research projects all around the world that use quadrotors for power line inspection. As stated in the dissertation of Ellis, in August 2013, in Australia alone, there were already 16 companies listed as licensed UAV operators for power line inspection [98]. We do not have details on the level of automation in these applications. However, the following projects clearly focus on automating power line inspection.

Li et al. [4] already perform fully-automated power line inspection. They use a helicopter of $31 \mathrm{~kg}$, which also carries enough fuel for up to one hour of flight. Furthermore, their helicopter is equipped with a more advanced sensor suite, which eases flight automation. Although promising, such platforms do not fit into the inexpensive category.

Several projects strongly focus on the image processing part of inspection, from various perspectives. Zhang et al. [99], for instance, compare some line detection algorithms for identifying and tracking power lines from videos captured by quadrotors. Luque-Vega et al. [100] combine a color camera with a thermal infrared camera to inspect infrastructure components. Larrauri et al. [101] deal with calculating distance to vegetation, trees and buildings, based on video frames. Martinez et al. [102] perform power line tower inspection. Their tracking approach steers the camera so as to keep an inspected tower in focus while flying along lines.

\subsection{Building Monitoring}

Another popular application field is the inspection of building façades and other surfaces, with the aim of examining their integrity. In such use-cases, the primary goal is to design flight plans that allow for proper data acquisition.

Baiocchi et al. [3] use quadrotors in a post-seismic environment for inspecting historic buildings for cracks and other damages. The GPS-based path planner developed by them optimizes flight paths, reducing redundant acquisition. Furthermore, their processing software allows for the 3D reconstruction of building façades from pairs of images. Another project, led by Eschmann et al. [103], presents a similar application, though with manual flight control.

Nikolic et al. [104] deal with power plant boiler inspection. They design an automated trajectory-following system and a personalized sensor suite for visual navigation. Using these, they examine the interior walls of boilers, expanding the GPS navigation functionality of the quadrotor with visual navigation, in order to be able to operate in GPS-denied regions, as well. 


\subsection{Railway Infrastructure Inspection}

Railway inspection comprises the tasks of structure inspection and linear structure following. In this context, railway inspection is related to the previous two fields. It is an application area not yet considered in public research projects, to the best of our knowledge. As presented below, newsletters report on several companies that intend to or already use UAVs in railways, based mainly on manual teleoperation or automated waypoint navigation. However, we have limited technical information on the work performed by these groups.

In the spring of 2013, news appeared about German Railways (DB - Deutsche Bahn) regarding their intention of using UAVs for catching graffiti sprayers [105]. French National Railways (SNCF - Société nationale des chemins de fer français) announced in the autumn of 2013 a project of railway bridge and viaduct inspection using UAVs [106]. The international company Elimco is also offering inspection of various infrastructures, including railways [107]. Similar applications can be found at Microdrones, where automated waypoint navigation is already implemented [108]. An article from Smartrail World reports on further companies that use or plan to work with UAVs: NetworkRail from the UK, ProRail from The Netherlands, Union Pacific from the USA and the Jerusalem light rail network [109]. Although few technical details are publicly available about these projects, they mainly seem to be based on manual teleoperation.

\subsection{Sensors in Infrastructure Inspection Applications}

Most papers discussed above provide generic or little information about the sensors used. In general, some kind of color cameras are used to perform data acquisition. From the works where more details are provided, Hausamann et al. [95] use a combination of optical and infrared sensors, discussing sensor types with different spectral bands; and synthetic aperture radars that have higher availability than color cameras due to the fact that radars are independent of weather and light conditions. Luque-Vega et al. [100] combine thermal-infrared cameras with color cameras for improving background subtraction and, thus, object detection. Larrauri et al. [101] use an HD camera in their project, though for processing data at only $4 \mathrm{fps}$. Eschmann et al. [103] perform navigation based on GPS data and use an external 12-MP commercial camera for offline post-processing only. Nikolic et al. [104] consider a CMOS image sensor and low-cost inertial sensors on a custom-built integrated circuit that aids UAV navigation and data collection in GPS-denied environments.

From these various details, one can conclude that color cameras are usually considered for data acquisition. Often, the performance of these cameras is reduced, but still good enough, while aiming at keeping the device low cost or at respecting payload constraints. Among the applications discussed, in certain cases, infrared cameras are additionally used to improve detection.

\section{Illustration in Railway Inspection Use-Cases}

Finally, we illustrate in this section the use of the camera-based sensing and control methods presented in Sections 2 and 3 for automated railway inspection. We formulate two use-cases: one where the UAV performs infrastructure inspection in close, but difficult-to-access areas (such as long bridges or tracks 
separated from the road by, e.g., a river). A second use-case is meant for railway track following for the sake of recording the infrastructure, such as tracks, sleepers, points or cabling. In the first use-case, the automated control runs on a remote station, while in the second one, full control is onboard. The chosen UAV platform is the Parrot AR.Drone 2.0 quadrotor, presented in Section 4. In certain cases, the implementation ideas come from the specifics of this particular platform, but they are usually valid for other low-cost quadrotors, as well.

We continue with evaluating the vision methods presented in Section 2, namely edge detectors for rail track detection in Section 6.1 and feature detectors for target (signal light) detection in Section 6.2. Then, we break down the use-cases into subtasks. We select detection solutions based on the results from our experiments detailed in Sections 6.1 and 6.2. Furthermore, we indicate other vision and control methods needed for our setup, based on the discussions from Sections 2 and 3. In this manner, our method selection from Section 6.3 considers, to an extent, our own experimental results and further solutions from the conclusions of our survey.

\subsection{Evaluating Feature Detectors for Target Detection}

In the first use-case, the quadrotor must find the target object before inspecting it. Considering that the target is close enough to the quadrotor (below $10 \mathrm{~m}$ ) and is in its field of view, one can apply feature detection, description and matching methods that, given a reference image of the target, can identify it in a new image, called the scene image.

Numerous detection, description and matching methods are readily implemented. We consider the OpenCV 2.4.9 library [110] that, from the methods discussed in Section 2.1, comes with implementations for the SURF, SIFT, FAST and Shi-Tomasi feature detectors and the SURF, SIFT, BRIEF and ORB descriptors. Each of these methods has a number of tuning parameters, as presented on the OpenCV website [110]. Based on prior tuning tests, we select a grid of meaningful values for these parameters (values marked with bold are the best according to the optimization criterion we describe below).

- For SURF, the Hessian threshold is taken in $\{300,310, \ldots, \mathbf{4 2 0}\}$, the number of octaves in $\{\mathbf{2}, 3, \ldots, 8\}$ and the number of octave layers in $\{1,2,3, \mathbf{4}, 5,6\}$, and we will use up-right features and extended descriptors.

- For SIFT, we allow all features to be retained, and we set the number of octaves in $\{1,2,3\}$, the contrast threshold in $\{0.01, \mathbf{0 . 0 2}, \ldots, 0.16\}$, the edge threshold in $\{5, \mathbf{7}, \ldots, 15\}$ and the standard deviation of the Gaussian in $\{1.2,1.3, \mathbf{1 . 4}, \ldots, 2.1\}$.

- For FAST, we use threshold values between $\{0,1, \ldots, \mathbf{3 1}, \ldots, 80\}$ and test both enabling and disabling non-max suppression.

- For Shi-Tomasi, we allow all features to be retained, set a quality level from $\{0.001,0.006, \ldots, \mathbf{0 . 0 2 1}, \ldots, 0.041\}$, a minimum feature distance of $\{0, \mathbf{1}, \ldots, 5\}$, an evaluation block size of $\{3,4,5, \mathbf{6}\}$, allow for enabling or disabling the Harris detector and set parameter $k$ to $\{0.02, \mathbf{0 . 0 4}, \ldots, 0.1\}$.

With each setting, we combine the detectors with all of the descriptors and a FLANN-based matcher [38] in order to evaluate the performance of the methods. The descriptors and the matcher require no parameter tuning. 
As the data source, we consider a $46 \times 188$ px reference image and 50 scene images, all $640 \times 360$ px in size and rectified. The reference image was taken with the same camera as the scene images. Each scene image contains the target object at different distances. Figure 3 shows a sample matching result.

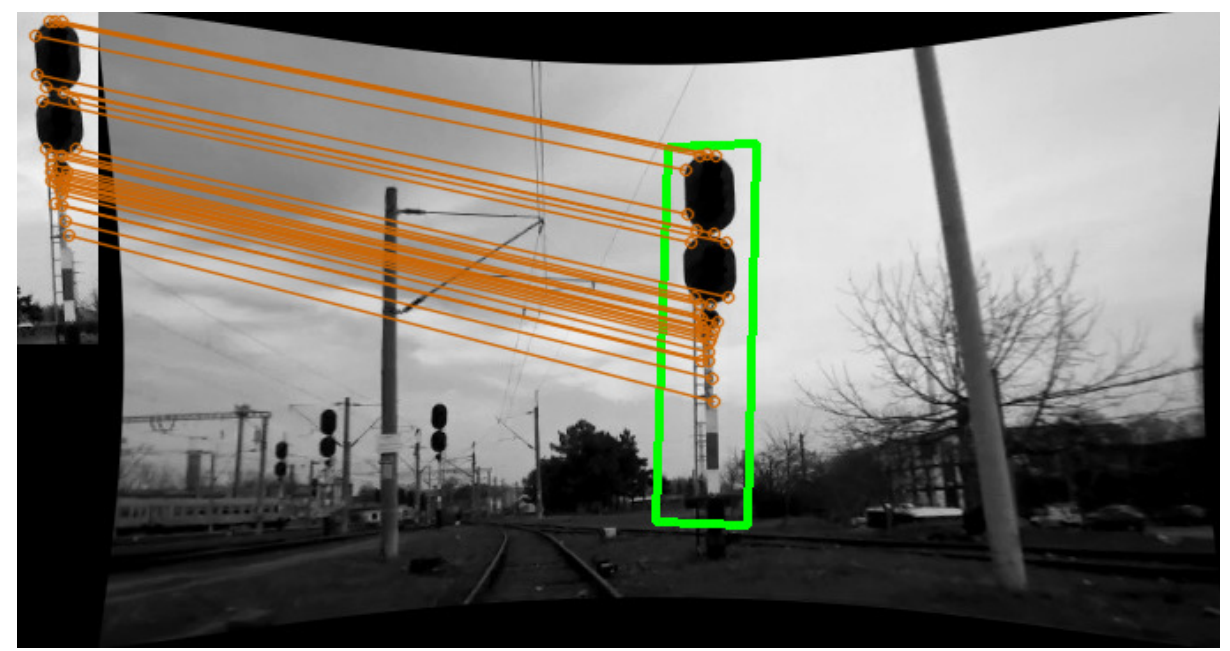

Figure 3. Target detection. Matching result with the reference and scene image.

After testing the various combinations of detector and descriptor methods, we obtain meaningful results only when using the SIFT descriptor. The other descriptors, in general, fail to detect the target object. Then, testing all of the detectors on the grid of parameters, we select the best parameter set for each detection algorithm that maximizes the detection rates during the simulations. Table 1 summarizes the performance of the four detectors in the case of these parameter sets.

Table 1. Performance evaluation of the feature detectors for target detection.

\begin{tabular}{ccccc}
\hline Method & Detect. Rate (\%) & Execution Time (ms) & Position Error $(\mathbf{p x})$ & Scaling Error (\%) \\
\hline SURF & 8 & 71.5 & 41.7 & 5 \\
SIFT & 54 & 17.4 & 2.7 & 45 \\
FAST & 98 & 8.3 & 2.2 & 37 \\
Shi-Tomasi & 96 & 7.4 & 2.2 & 41 \\
\hline
\end{tabular}

As shown in Table 1, we evaluate the detection success rate, the average execution time per frame and average errors on the horizontal position and the scaling of the detected object. The detection rate tells in how many frames the algorithm detected the reference object. The average execution time considers the total time required for detection, description and matching, for a single frame, i.e., the total image processing time. The average position error tells the horizontal distance in pixels between the real center of the object in the scene image and the center of the detected area. Finally, the scaling error indicates the average difference between the size of the reference image and the size of the detected object, expressed in percentages, i.e., it is a size detection error. Note that the latter two parameters indicate the accuracy of the detection, while the first two inform about the usefulness of the algorithm in the case of online use.

According to the results from Table 1, the FAST and Shi-Tomasi methods outperform the other two, both in detection rate and execution time. The detection rate of SIFT is also acceptable. Looking at 
the average position error, all of the methods perform well, except SURF. However, the scaling error indicates the opposite. SURF, although it has a far lower detection rate, detects the object size more precisely. Furthermore, higher scaling errors in the case of the other methods appear since the object detection algorithm was implicitly allowed to have these errors in favor of higher detection rates by the optimization procedure. Additionally, the small position errors indicate that, with the last three methods, the target was identified in the correct place.

Based on these results, we select the FAST algorithm for target detection in the given use-case, in combination with the SIFT descriptor. However, we remark that the parameter sets were fine-tuned for the given dataset, and other combinations might turn out to have better results for different scenarios where other reference/target objects or other image parameters are considered. Nevertheless, we highlight the good performance of the FAST and Shi-Tomasi detectors, which, with an average execution time below $10 \mathrm{~ms}$, allow for at least 100-Hz control, good enough for target detection during flight with a quadrotor. With respect to the discussion in Section 2.1, the selection of the Shi-Tomasi detector confirms the conclusions from there. However, the results of the FAST detector are even better in our particular setup, while the Harris detector seems to reduce the performance when applied in the Shi-Tomasi algorithm.

\subsection{Evaluating Edge Detectors for Track Detection}

In the second use-case, the quadrotor has to follow track lines. This can be accomplished by vanishing point-based control, as introduced briefly below in Section 6.3.1 and presented in [111]. To achieve this aim, edge detector methods can be used in combination with line extractors that help with finding the track lines, which are then used to find their vanishing point.

From the edge detectors mentioned in Section 2.1, OpenCV comes with implementations for Canny, Sobel, Laplacian and Scharr algorithms. We will test these methods on a grid of meaningful parameter sets and combine them with a probabilistic Hough transform (PHT) for line extraction and a custom filtering method for line selection. This method removes all of the lines up to a vertical angle, after which it progressively filters out the lines that do not point to an average vanishing point. An example of the processed image and obtained lines and vanishing point is shown in Figure 4.

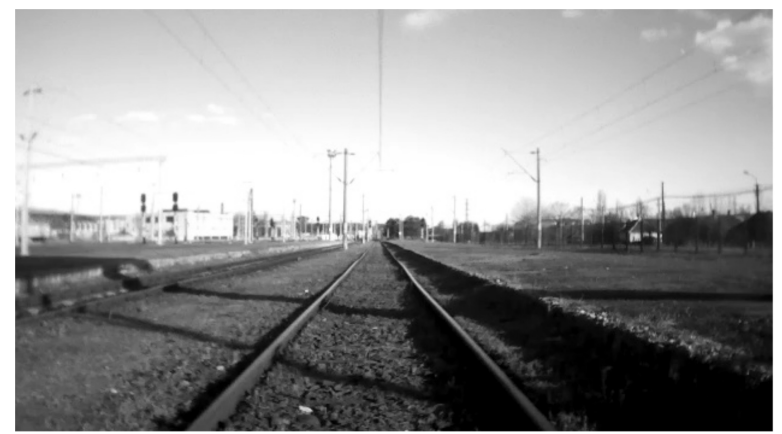

(a)

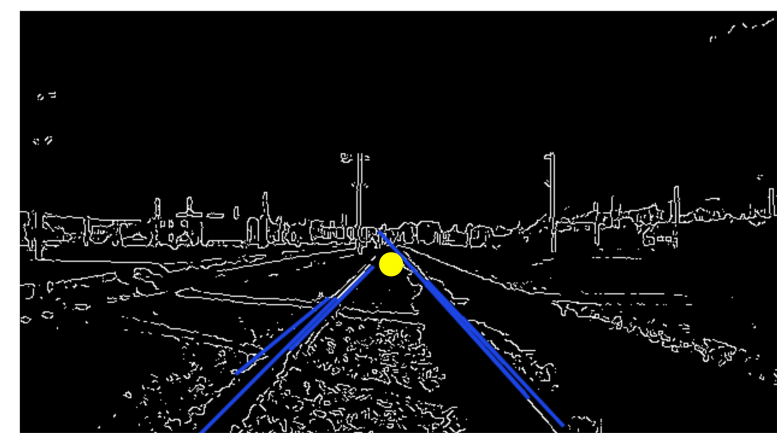

(b)

Figure 4. (a) Track line detection; (b) Canny edge detection result and vanishing point detection. 
The parameters of the detectors are taken according to the following grid (best values marked with bold or stated separately).

- For all of the methods, we worked with default image color depth and edge modeling border type and set the $x$ and $y$ derivative orders in $\{0,1,2\}$, kernel sizes in $\{1,3,5,7\}$, derivative scaling factors in $\{0.001,0.002, \ldots, 0.01,0.015, \ldots, 0.14\}$ and a delta value, added to the pixels of the output image during the convolution, in $\{0,0.01, \ldots, 0.40\}$. Note that some parameters appear with only some of the methods.

- In the case of Canny, the two hysteresis thresholds are taken in the interval $\{0,10, \ldots, 90,91, \ldots, \mathbf{1 0 6}, \ldots ., 110,120, \ldots ., 300\}$; the best kernel size was 3 , and we allowed for enabling or disabling the use of the $L_{2}$ gradient in image gradient magnitude calculation.

- For Sobel, the best values of the parameters are: derivative orders $x=1$ and $y=0$, kernel size three, scaling factor 0.0125 and delta 0.037 .

- For Laplacian, the best values are: kernel size five, scaling factor 0.002 and delta 0.095 .

- For Scharr, the best values are: derivative orders $x=1$ and $y=0$, scaling factor 0.001 and delta 0.23 .

From the common parameters, the kernel size is the size of the matrix used in calculating the transformed image. In our experiments, we observe that keeping this value low provides better results. The scaling factor determines the image dimming, and better results are obtained when keeping this value low, i.e., having almost completely dimmed images. The delta value has no visible influence on the image processing, although it turns out that the lower its value, the better the detection.

We applied these detectors for a set of 165 scene images of size $640 \times 360 \mathrm{px}$, all containing a pair of track lines with different orientations, after which the line extraction and selection algorithms were executed. We evaluated the average execution time and the average and maximum position errors. The execution time is calculated per frame, for the detection algorithms only, as these are the subject of our evaluation. The position errors determine the difference in pixels between the real (ground truth) and measured horizontal position of the vanishing point of the tracks. Based on these indicators, Table 2 summarizes the performance of the detection methods for the parameter sets for which the average position error was the lowest.

Table 2. Performance evaluation of edge detectors for line detection.

\begin{tabular}{cccc}
\hline Method & Exec Time (ms) & Average Position Error $(\mathbf{p x})$ & Max Positioning Error $(\mathbf{p x})$ \\
\hline Canny & 1.60 & 17.5 & 126 \\
Sobel & 2.27 & 16.4 & 193 \\
Laplacian & 2.74 & 13.8 & 79 \\
Scharr & 2.19 & 16.9 & 193 \\
\hline
\end{tabular}

From Table 2, one can see that all of the methods have an execution time below $3 \mathrm{~ms}$. Recall that we considered only the duration of the detection, which together with the line extraction and selection results in times up to $20-25 \mathrm{~ms}$. Still, this offers a $40-50-\mathrm{Hz}$ control rate in the case of any detection algorithm, 
high enough for quadrotor control. The maximum position error indicates some false detections, which is the least severe in the case of the Laplacian algorithm. However, given the $640 \mathrm{px}$ image width, all of the methods have an average position error below $2.5 \%$ that indicates an overall correct detection. From all of these parameters, we prefer to consider the position error the most important and use, therefore, the Laplacian method in our use-case of track following. Nevertheless, the test results confirm the discussion from Section 2.1 on the good performance of the Canny detector and its weakness of generating false positives, when comparing with the performance of the Laplacian method.

\subsection{Use-Case Subtasks}

We select solutions for the two use-cases, the short-range inspection in difficult-to-access areas and the long-range, track following-based infrastructure recording. This selection is just an illustration of how the presented methods can be used for UAV navigation. We provide no details on the settings of the considered techniques. However, flight tests were also already performed that demonstrate the suitability of several selected methods.

First, we need to break down the use-cases into subtasks. These are mainly: take-off, fly on a path, find and inspect targets, fly home and land. Next, we detail these subtasks and propose solutions to the related control problems. The take-off and landing tasks are, in the case of the AR.Drone and with many other RTF quadrotors, already solved by built-in functions. Furthermore, the newest products come with a fly-home function that, based on GPS coordinates, makes the quadrotor return autonomously to its take-off location and land there. For automation of the other tasks, additional processing and control methods are required.

\subsubsection{Flying on a Path}

Flying on a path poses different challenges in the two use-cases. In the case of remotely-controlled local inspection, it can be solved by GPS waypoint navigation with obstacle avoidance. Here, three subtasks can be identified: planning the waypoint sequence, navigating using GPS data and obstacle detection and avoidance. The waypoints have to be planned, e.g., to optimize the flight time or to avoid obstacles. For online planning, we suggest the use of the RRT and MPC-based algorithms. Regarding GPS-based waypoint navigation, quadrotors with a GPS module like the AR.Drone usually have implementations for this task. We will consider the software from [112]. Finally, obstacle avoidance is one of the most challenging tasks for quadrotors. Here, based on the experiments from Section 6.1, we suggest using Laplacian filtering for detection, combined with optical flow techniques that can determine motion. Then, we recommend the previously mentioned online planning methods for the avoidance maneuver.

In the second use-case, railway following, the path planning and following problem boils down to line following. With an onboard camera looking ahead, this can be achieved, for example, by finding and tracking the vanishing point of the lines formed by the pair of rails [111]. The vanishing point detection consists of image preprocessing for edge detection, line detection and line filtering in order to identify the tracks and their vanishing point. Based on [111] and on our experiments from Section 6.2, we propose the use of the Laplacian operator for edge detection. Then, the probabilistic Hough transform (PHT) can 
be applied to find lines from the resulting contours. These lines can then be filtered simply based on their lengths and slopes: we select long enough lines (e.g., longer than a quarter of the image height) that are almost vertical. These lines have a vanishing point that matches the vanishing point of the tracks. The tracking subtask can be solved by simple visual servoing: the vanishing point can be kept in the middle of the camera image through a proportional derivative controller. More precisely, we propose to perform yaw ( $z$ axis) angle rotations, while additionally correcting with lateral displacement and forward velocity reduction if the vanishing point is outside the desired range.

\subsubsection{Finding and Inspecting Targets}

For both use-cases, a method to find the target and a navigation strategy are needed, whereas in the first use-case (local inspection), a further navigation solution is needed for inspection. We consider a database of images of the possible targets. Then, based on the conclusions from Section 6.1, we propose to use FAST feature detectors with SIFT descriptors and the FLANN-based matcher. Together, these methods can track an object in subsequent frames. Above a threshold for the detection rate over time, we consider the target being found. We also point out the solution presented in [102], where machine learning is used in combination with edge detectors to find the target using a database of reference images. Yet another idea is to test RANSAC model fitting, introduced in Section 2.1.

To navigate around a detected target, we consider visual servoing techniques. An alternative would be GPS-based navigation, although for the AR.Drone, the GPS accuracy is of $2 \mathrm{~m} \mathrm{[113],} \mathrm{not} \mathrm{enough}$ for safe navigation close to objects. We propose therefore the use of PBVS methods, introduced in Section 2.3. Furthermore, based on our experiments from Section 6.1, a basic visual servoing solution is to use the target detection described above together with homography-based identification. The obtained homography can then be used to determine the scaling and image-frame position of the target. Knowing the distance to the target in the reference image, the scaling and image-frame position can be then transformed into longitudinal and lateral distances to the object, which indicate the relative position to the target. Based on this, simple controllers can be applied to correct the distances so as to track the desired inspection trajectories.

\section{Summary and Outlook}

In the first major part of this paper, we reviewed vision and control methods for UAVs, with the final goal of using them in railway inspection applications. In the second part, we presented several popular low-cost quadrotor platforms, overviewed research concerning UAV inspection applications and formulated two use-cases. The use-cases address the novel application field of railway inspection and focus on short-range inspection in difficult-to-access areas and long-range track following. We performed an exhaustive evaluation of feature detectors for track following and target detection. Finally, we devised a strategy to accomplish the use-case task using results from our experiments and from the conclusions of the survey.

The survey of vision and control techniques revealed several open challenges, like the difficult problem of fine-tuning in the case of vision methods, the high computational demands of both vision and flight planning tools compared to the onboard processing capacity of the low-cost UAVs or the limitations 
appearing due to the lack of adequate mission formalization and due to the restrictive regulations. Further open issues are the lack of a general obstacle avoidance solution, which is crucial for fully-automated UAV navigation, and limitations derived from the short battery life of low-cost UAVs. Our future work is motivated by the railway inspection use-cases, and we are currently continuing our research by evaluating additional vision techniques for object classification and for obstacle avoidance, by developing trajectory and path planning techniques for automated flight of low-cost quadrotors.

\section{Acknowledgments}

This paper is supported by a grant from Siemens, Reference No. 7472/3202246859; by the Sectoral Operational Programme Human Resources Development (SOP HRD), ID 137516 (POSDRU/159/1.5/S/137516), financed by the European Social Fund and by the Romanian Government; and by a grant from the Romanian National Authority for Scientific Research, Project Number PNII-RU-TE-2012-3-0040.

We are grateful to Péter Virág from University of Miskolc, Hungary, for his contribution regarding the testing of image processing techniques; and to Cristea-Ioan Iuga from Technical University of Cluj-Napoca, Romania, for his help in performing the flight tests and data collection.

\section{Author Contributions}

Koppány Máthé is the main author, having conducted the survey and written the content. Lucian Buşoniu has contributed with numerous comments, as well as content in the Introduction and Abstract.

\section{Conflicts of Interest}

The authors declare no conflict of interest.

\section{References}

1. Bakx, G.; Nyce, J. UAS in the (Inter)national airspace: Approaching the debate from an ethnicity perspective. In Proceedings of the International Conference on Unmanned Aircraft Systems (ICUAS), Atlanta, GA, USA, 28-31 May 2013; pp. 189-192.

2. DJI Products. Available online: http://www.dji.com/products (accessed on 22 December 2014).

3. Baiocchi, V.; Dominici, D.; Mormile, M. UAV application in post-seismic environment. Int. Arch. Photogramm. Remote Sens. Spatial Inf. Sci. XL-1 W 2013, 2, 21-25.

4. Li, H.; Wang, B.; Liu, L.; Tian, G.; Zheng, T.; Zhang, J. The design and application of SmartCopter: An unmanned helicopter based robot for transmission line inspection. In Proceedings of the IEEE Chinese Automation Congress (CAC), Changsha, China, 7-8 November 2013; pp. 697-702.

5. Parrot AR.Drone. Available online: http://ardrone2.parrot.com/ar-drone-2/specifications/ (accessed on 22 December 2014). 
6. ArduCopter Quadrocopter. Available online: http://www.uav-store.de/diy-kits/3dr-quadrocopter (accessed on 22 December 2014).

7. Grabe, V.; Bulthoff, H.; Giordano, P. On-board Velocity Estimation and Closed-loop Control of a Quadrotor UAV based on Optical Flow. In Proceedings of the IEEE International Conference on Robotics and Automation (ICRA), St Paul, MN, USA, 14-18 May 2012; pp. 491-497.

8. Dydek, Z.; Annaswamy, A.; Lavretsky, E. Adaptive Control of Quadrotor UAVs: A Design Trade Study with Flight Evaluations. IEEE Trans. Control Syst. Technol. 2013, 21, 1400-1406.

9. Pestana, J.; Mellado-Bataller, I.; Sanchez-Lopez, J.L.; Fu, C.; Mondragón, I.F.; Campoy, P. A General Purpose Configurable Controller for Indoors and Outdoors GPS-Denied Navigation for Multirotor Unmanned Aerial Vehicles. J. Intell. Robotic Syst. 2014, 73, 387-400.

10. Fossel, J.; Hennes, D.; Claes, D.; Alers, S.; Tuyls, K. OctoSLAM: A 3D mapping approach to situational awareness of unmanned aerial vehicles. In Proceedings of the International Conference on Unmanned Aircraft Systems (ICUAS), Atlanta, GA, USA, 28-31 May 2013; pp. 179-188.

11. Stark, B.; Smith, B.; Chen, Y. Survey of thermal infrared remote sensing for Unmanned Aerial Systems. In Proceedings of the IEEE International Conference on Unmanned Aircraft Systems (ICUAS), Orlando, FL, USA, 27-30 May 2014; pp. 1294-1299.

12. Bendig, J.; Bolten, A.; Bareth, G. Introducing a low-cost mini-UAV for thermal-and multispectral-imaging. Int. Arch. Photogramm. Remote Sens. Spat. Inf. Sci. 2012, 39, 345-349.

13. Wenzel, K.E.; Masselli, A.; Zell, A. Automatic take off, tracking and landing of a miniature UAV on a moving carrier vehicle. J. Intell. Robot. Syst. 2011, 61, 221-238.

14. Whitehead, K.; Hugenholtz, C.H. Remote sensing of the environment with small unmanned aircraft systems (UASs), part 1: A review of progress and challenges 1. J. Unmanned Veh. Syst. 2014, 2, 69-85.

15. Baker, S.; Scharstein, D.; Lewis, J.; Roth, S.; Black, M.J.; Szeliski, R. A database and evaluation methodology for optical flow. Int. J. Comput. Vis. 2011, 92, 1-31.

16. Oskoei, M.A.; Hu, H. A Survey on Edge Detection Methods; University of Essex: Essex, UK, 2010.

17. Hua, M.D.; Hamel, T.; Morin, P.; Samson, C. Introduction to Feedback Control of Underactuated VTOL Vehicles. IEEE Control Syst. Mag. 2013, 33, 61-75.

18. Goerzen, C.; Kong, Z.; Mettler, B. A survey of motion planning algorithms from the perspective of autonomous UAV guidance. J. Intell. Robot. Syst. 2010, 57, 65-100.

19. Dadkhah, N.; Mettler, B. Survey of motion planning literature in the presence of uncertainty: Considerations for UAV guidance. J. Intell. Robot. Syst. 2012, 65, 233-246.

20. LaValle, S.M. Planning Algorithms; Cambridge University Press: Cambridge, UK, 2006.

21. Roushdy, M. Comparative study of edge detection algorithms applying on the grayscale noisy image using morphological filter. GVIP J. 2006, 6, 17-23.

22. Tuytelaars, T.; Mikolajczyk, K. Local invariant feature detectors: A survey. Found. Trends Comput. Graph. Vis. 2008, 3, 177-280.

23. Choi, S.; Kim, T.; Yu, W. Performance Evaluation of RANSAC Family. In Proceedings of the British Machine Vision Conference (BMVC 2009), British Machine Vision Association (BMVA), London, UK, 7-10 September 2009; pp. 81.1-81.12. 
24. Rockett, P. Performance assessment of feature detection algorithms: A methodology and case study on corner detectors. IEEE Trans. Image Proc. 2003, 12, 1668-1676.

25. Jain, R.; Kasturi, R.; Schunck, B.G. Machine Vision; McGraw-Hill: New York, NY, USA, 1995.

26. Rufeil, E.; Gimenez, J.; Flesia, A. Comparison of edge detection algorithms on the undecimated wavelet transform. In Proceedings of the IV CLAM, Latin American Congress in Mathematics, Córdoba, Argentina, 6-10 August 2012.

27. Senthilkumaran, N.; Rajesh, R. Edge detection techniques for image segmentation-A survey of soft computing approaches. Int. J. Recent Trends Eng. 2009, 1, 250-254.

28. Harris, C.; Stephens, M. A combined corner and edge detector. In Proceedings of the Alvey Vision Conference, Manchester, UK, 31 August-2 September 1988; Volume 15, p. 50.

29. Shi, J.; Tomasi, C. Good features to track. In Proceedings of the IEEE Computer Society Conference on Computer Vision and Pattern Recognition (CVPR'94), Seattle, WA, USA, 21-23 June 1994; pp. 593-600.

30. Tulpan, D.; Belacel, N.; Famili, F.; Ellis, K. Experimental evaluation of four feature detection methods for close range and distant airborne targets for Unmanned Aircraft Systems applications. In Proceedings of the IEEE International Conference on Unmanned Aircraft Systems (ICUAS), Orlando, FL, USA, 27-30 May 2014; pp. 1267-1273.

31. Smith, S.M.; Brady, J.M. SUSAN-A new approach to low level image processing. Int. J. Comput. Vis. 1997, 23, 45-78.

32. Rosten, E.; Drummond, T. Rapid rendering of apparent contours of implicit surfaces for real-time tracking. In Proceedings of the 14th British Machine Vision Conference (BMVC 2003), British Machine Vision Association (BMVA), Norwich, UK, 9-11 September 2003; pp. 719-728.

33. Bay, H.; Tuytelaars, T.; van Gool, L. Surf: Speeded up robust features. In Computer Vision-ECCV 2006; Springer: Berlin/Heidelberg, Germany, 2006; Volume 3951, pp. 404-417.

34. Lowe, D.G. Distinctive image features from scale-invariant keypoints. Int. J. Comput. Vis. 2004, 60, 91-110.

35. Calonder, M.; Lepetit, V.; Strecha, C.; Fua, P. Brief: Binary robust independent elementary features. In Computer Vision -ECCV 2010; Springer: Berlin/Heidelberg, Germany, 2010; Volume 6314, pp. 778-792.

36. Rublee, E.; Rabaud, V.; Konolige, K.; Bradski, G. ORB: An efficient alternative to SIFT or SURF. In Proceedings of the IEEE International Conference on Computer Vision (ICCV), Barcelona, Spain, 6-13 November 2011; pp. 2564-2571.

37. Arya, S.; Mount, D.M.; Netanyahu, N.S.; Silverman, R.; Wu, A.Y. An optimal algorithm for approximate nearest neighbor searching fixed dimensions. J. ACM 1998, 45, 891-923.

38. Muja, M.; Lowe, D.G. Fast matching of binary features. In Proceedings of the IEEE Ninth Conference on Computer and Robot Vision (CRV), Toronto, ON, Canada, 28-30 May 2012; pp. 404-410.

39. Dubrofsky, E. Homography Estimation. Ph.D Thesis, University of British Columbia (Vancouver), Kelowna, BC, Canada, March 2009. 
40. Zsedrovits, T.; Bauer, P.; Zarándy, A.; Vanek, B.; Bokor, J.; Roska, T. Error Analysis of Algorithms for Camera Rotation Calculation in GPS/IMU/Camera Fusion for UAV Sense and Avoid Systems. In Proceedings of the IEEE International Conference on Unmanned Aircraft Systems (ICUAS), Orlando, FL, USA, 27-30 May 2014.

41. Flores, G.; Zhou, S.; Lozano, R.; Castillo, P. A Vision and GPS-Based Real-Time Trajectory Planning for a MAV in Unknown and Low-Sunlight Environments. J. Intell. Robot. Syst. 2014, 74, 59-67.

42. Yang, S.; Scherer, S.A.; Schauwecker, K.; Zell, A. Autonomous Landing of MAVs on an Arbitrarily Textured Landing Site Using Onboard Monocular Vision. J. Intell. Robot. Syst. 2014, 74, 27-43.

43. Schauwecker, K.; Zell, A. On-Board Dual-Stereo-Vision for the Navigation of an Autonomous MAV. J. Intell. Robot. Syst. 2014, 74, 1-16.

44. Herout, A.; Dubská, M.; Havel, J. Review of Hough Transform for Line Detection. In Real-Time Detection of Lines and Grids; Springer: Berlin/Heidelberg, Germany, 2013; Volume 1, pp. 3-16.

45. Mukhopadhyay, P.; Chaudhuri, B.B. A survey of Hough Transform. Pattern Recog. 2015, 48, 993-1010.

46. OpenCV Probabilistic Hough Transform implementation. Available online: http://docs.opencv. org/modules/imgproc/doc/feature_detection.html (accessed on 8 May 2015).

47. Chao, H.; Gu, Y.; Napolitano, M. A Survey of Optical Flow Techniques for Robotics Navigation Applications. J. Intell. Robot. Syst. 2014, 73, 361-372.

48. Sun, D.; Roth, S.; Black, M.J. Secrets of optical flow estimation and their principles. In Proceedings of the IEEE Conference on Computer Vision and Pattern Recognition (CVPR), San Francisco, CA, USA, 13-18 June 2010; pp. 2432-2439.

49. Sun, D.; Roth, S.; Black, M.J. A quantitative analysis of current practices in optical flow estimation and the principles behind them. Int. J. Comput. Vis. 2014, 106, 115-137.

50. Dame, A.; Marchand, E. Mutual information-based visual servoing. IEEE Trans. Robot. 2011, 27, 958-969.

51. Caron, G.; Dame, A.; Marchand, E. Direct model based visual tracking and pose estimation using mutual information. Image Vis. Comput. 2014, 32, 54-63.

52. Collewet, C.; Marchand, E. Photometric visual servoing. IEEE Trans. Robot. 2011, 27, 828-834.

53. Thuilot, B.; Martinet, P.; Cordesses, L.; Gallice, J. Position based visual servoing: Keeping the object in the field of vision. In Proceedings of the IEEE International Conference on Robotics and Automation ( ICRA '02), Washington, DC, USA, 11-15 May 2002; Volume 2, pp. 1624-1629.

54. Chaumette, F.; Malis, E. 2 1/2 D visual servoing: A possible solution to improve image-based and position-based visual servoings. In Proceedings of the IEEE International Conference on Robotics and Automation ( ICRA'00), San Francisco, CA, USA, 24-28 April 2000; Volume 1, pp. 630-635.

55. Bourquardez, O.; Mahony, R.; Guenard, N.; Chaumette, F.; Hamel, T.; Eck, L. Image-Based Visual Servo Control of the Translation Kinematics of a Quadrotor Aerial Vehicle. IEEE Trans. Robot. 2009, 25, 743-749. 
56. Fahimi, F.; Thakur, K. An alternative closed-loop vision-based control approach for Unmanned Aircraft Systems with application to a quadrotor. In Proceedings of the IEEE International Conference on Unmanned Aircraft Systems (ICUAS), Atlanta, GA, USA, 28-31 May 2013; pp. 353-358.

57. Bai, Y.; Liu, H.; Shi, Z.; Zhong, Y. Robust control of quadrotor unmanned air vehicles. In Proceedings of the IEEE 31st Chinese Control Conference (CCC), Hefei, China, 25-27 July 2012; pp. 4462-4467.

58. Krajnik, T.; Nitsche, M.; Pedre, S.; Preucil, L.M.A. A simple visual navigation system for an UAV. In Proceedings of the 9th International Multi-Conference on Systems, Signals and Devices (SSD), Chemnitz, Germany, 20-23 March 2012; pp. 1-6.

59. Ross, S.; Melik-Barkhudarov, N.; Shankar, K.S.; Wendel, A.; Dey, D.; Bagnell, J.A.; Hebert, M. Learning monocular reactive uav control in cluttered natural environments. In Proceedings of the IEEE International Conference on Robotics and Automation (ICRA), Karlsruhe, Germany, 6-10 May 2013; pp. 1765-1772.

60. Amelink, M.H.J. Ecological Automation Design, Extending Work Domain Analysis. Ph.D Thesis, Delft University of Technology, Delft, Holland, October 2010.

61. García Carrillo, L.R.; Dzul López, A.E.; Lozano, R.; Pégard, C. Modeling the Quad-Rotor Mini-Rotorcraft. In Quad Rotorcraft Control; Springer: London, UK, 2013; Volume 1, pp. 23-34.

62. Salazar, S.; Gonzalez-Hernandez, I.; López, J.R.; Lozano, R.; Romero, H. Simulation and Robust Trajectory-Tracking for a Quadrotor UAV. In Proceedings of the IEEE International Conference on Unmanned Aircraft Systems (ICUAS), Orlando, FL, USA, 27-30 May 2014.

63. Cui, Y.; Inanc, T. Controller design for small air vehicles-An overview and comparison. In Proceedings of the IEEE International Conference on Unmanned Aircraft Systems (ICUAS), Atlanta, GA, USA, 28-31 May 2013; pp. 621-627.

64. Raffo, G.V.; Ortega, M.G.; Rubio, F.R. An integral predictive/nonlinear HấLđ control structure for a quadrotor helicopter. Automatica 2010, 46, 29-39.

65. Alexis, K.; Nikolakopoulos, G.; Tzes, A. On trajectory tracking model predictive control of an unmanned quadrotor helicopter subject to aerodynamic disturbances. Asian J. Control 2014, 16, 209-224.

66. Lin, Y.; Saripalli, S. Path planning using 3D Dubins Curve for Unmanned Aerial Vehicles. In Proceedings of the IEEE International Conference on Unmanned Aircraft Systems (ICUAS), Orlando, FL, USA, 27-30 May 2014; pp. 296-304.

67. Bellingham, J.; Richards, A.; How, J.P. Receding horizon control of autonomous aerial vehicles. In Proceedings of the IEEE 2002 American Control Conference, Anchorage, AK, USA, 8-10 May 2002; Volume 5, pp. 3741-3746.

68. Chao, Z.; Zhou, S.L.; Ming, L.; Zhang, W.G. Uav formation flight based on nonlinear model predictive control. Math. Probl. Eng. 2012, 2012, doi:10.1155/2012/261367

69. Grancharova, A.; Grøtli, E.I.; Ho, D.T.; Johansen, T.A. UAVs Trajectory Planning by Distributed MPC under Radio Communication Path Loss Constraints. J. Intell. Robot. Syst. 2014, 1-20, doi:10.1007/s10846-014-0090-1. 
70. Donald, B.; Xavier, P.; Canny, J.; Reif, J. Kinodynamic motion planning. J. ACM 1993, 40, 1048-1066.

71. Franze, G.; Mattei, M.; Ollio, L.; Scordamaglia, V. A Receding Horizon Control scheme with partial state measurements: Control Augmentation of a flexible UAV. In Proceedings of the IEEE Conference on Control and Fault-Tolerant Systems (SysTol), Nice, France, 9-11 October 2013; pp. $158-163$.

72. Sahingoz, O.K. Generation of Bezier Curve-Based Flyable Trajectories for Multi-UAV Systems with Parallel Genetic Algorithm. J. Intell. Robot. Syst. 2014, 74, 499-511.

73. Schopferer, S.; Adolf, F.M. Rapid trajectory time reduction for unmanned rotorcraft navigating in unknown terrain. In Proceedings of the IEEE International Conference on Unmanned Aircraft Systems (ICUAS), Orlando, FL, USA, 27-30 May 2014; pp. 305-316.

74. Zufferey, J.C.; Beyeler, A.; Floreano, D. Autonomous flight at low altitude with vision-based collision avoidance and GPS-based path following. In Proceedings of the IEEE International Conference on Robotics and Automation (ICRA), Anchorage, AK, USA, 3-8 May 2010; pp. 3329-3334.

75. Qu, Y.; Zhang, Y.; Zhang, Y. Optimal Flight Path Planning for UAVs in 3-D Threat Environment. In Proceedings of the IEEE International Conference on Unmanned Aircraft Systems (ICUAS), Orlando, FL, USA, 27-30 May 2014.

76. Gimenes, R.A.; Vismari, L.F.; Avelino, V.F.; Camargo, J.B., Jr.; de Almeida, J.R., Jr.; Cugnasca, P.S. Guidelines for the Integration of Autonomous UAS into the Global ATM. J. Intell. Robot. Syst. 2014, 74, 465-478.

77. Faughnan, M.; Hourican, B.; MacDonald, G.; Srivastava, M.; Wright, J.; Haimes, Y.; Andrijcic, E.; Guo, Z.; White, J. Risk analysis of Unmanned Aerial Vehicle hijacking and methods of its detection. In Proceedings of the IEEE Systems and Information Engineering Design Symposium (SIEDS), Charlottesville, VA, USA, 26 April 2013; pp. 145-150.

78. RCgroups Community. Available online: http://www.rcgroups.com/forums/index.php (accessed on 11 May 2015).

79. Diydrones Community. Available online: http://diydrones.com/, (accessed on 11 May 2015).

80. My First Drone Website. Available online: http://myfirstdrone.com/ (accessed on 11 May 2015).

81. Drones for Good Website. Available online: https://www.dronesforgood.ae/en (accessed on 11 May 2015).

82. Best Quadcopters Website. Available online: http://www.bestquadcoptersreviews.com/ (accessed on 11 May 2015).

83. Parrot AR.Drone Pressure Sensor. Available online: http://ardrone2.parrot. com/ardrone-2/altitude (accessed on 22 December 2014).

84. Parrot Bebop Drone. Available online: http://www.parrot.com/products/bebop-drone/ (accessed on 11 May 2015).

85. Xaircraft Products. Available online: http://xaircraftamerica.com/collections/xaircraft-products/ products/x650-pro-kit-1 (accessed on 22 December 2014).

86. Xaircraft SuperX Flight Controller. Available online: http://www.xaircraft.com/products/superx/ (accessed on 22 December 2014). 
87. MikroKopter Products. Available online: http://www.quadrocopter.com/ MK-Basicset-QuadroKopter-XL_p_283.html (accessed on 22 December 2014).

88. MikroKopter Flight Controller. Available online: http://wiki.mikrokopter.de/en/FlightCtrl_ME_ 2_5 (accessed on 22 December 2014).

89. DJI Flight Controllers. Available online: http://www.dji.com/info/spotlight/whats-difference -of-naza-m-litenaza-m-v1naza-m-v2 (accessed on 22 December 2014).

90. ArduCopter Programming. Available online: http://copter.ardupilot.com/wiki/ programming-arducopter-with-arduino/ (accessed on 22 December 2014).

91. ArduCopter Irist. Available online: http://store.3drobotics.com/products/iris (accessed on 22 December 2014).

92. 3DR Solo. Available online: http://3drobotics.com/solo/ (accessed on 11 May 2015).

93. PIXHAWK Autopilot. Available online: http://pixhawk.org/modules/pixhawk (accessed on 22 December 2014).

94. PIXHAWK PX4FLOW. Available online: https://pixhawk.org/modules/px4flow (accessed on 22 December 2014).

95. Hausamann, D.; Zirnig, W.; Schreier, G.; Strobl, P. Monitoring of gas pipelines-A civil UAV application. Aircraft Eng. Aerosp. Technol. 2005, 77, 352-360.

96. Rathinam, S.; Kim, Z.W.; Sengupta, R. Vision-Based Monitoring of Locally Linear Structures Using an Unmanned Aerial Vehicle 1. J. Infrastruct. Syst. 2008, 14, 52-63.

97. Montambault, S.; Beaudry, J.; Toussaint, K.; Pouliot, N. On the application of VTOL UAVs to the inspection of power utility assets. In Proceedings of the IEEE 1st International Conference on Applied Robotics for the Power Industry (CARPI), Montreal, QC, Canada, 5-7 October 2010; pp. $1-7$.

98. Ellis, N. Inspection of Power Transmission Lines Using UAVs. Master's Thesis, University of Southern Queensland, Toowoomba, Queensland, Australia, October 2013.

99. Zhang, J.; Liu, L.; Wang, B.; Chen, X.; Wang, Q.; Zheng, T. High speed automatic power line detection and tracking for a UAV-based inspection. In Proceedings of the IEEE International Conference on Industrial Control and Miscs Engineering (ICICEE), Xi'an, China, 23-25 April 2012; pp. 266-269.

100. Luque-Vega, L.F.; Castillo-Toledo, B.; Loukianov, A.; Gonzalez-Jimenez, L.E. Power line inspection via an unmanned aerial system based on the quadrotor helicopter. In Proceedings of the IEEE 17th Mediterranean Electrotechnical Conference (MELECON), Beirut, Lebanon, 13-16 April 2014; pp. 393-397.

101. Larrauri, J.; Sorrosal, G.; González, M. Automatic system for overhead power line inspection using an Unmanned Aerial Vehicle - RELIFO project. In Proceedings of the IEEE International Conference on Unmanned Aircraft Systems (ICUAS), Atlanta, GA, USA, 28-31 May 2013; pp. 244-252.

102. Martinez, C.; Sampedro, C.; Chauhan, A.; Campoy, P. Towards autonomous detection and tracking of electric towers for aerial power line inspection. In Proceedings of the IEEE International Conference on Unmanned Aircraft Systems (ICUAS), Orlando, FL, USA, 27-30 May 2014; pp. 284-295. 
103. Eschmann, C.; Kuo, C.; Kuo, C.; Boller, C. Unmanned aircraft systems for remote building inspection and monitoring. In Proceedings of the Sixth European Workshop on Structural Health Monitoring, Dresden, Germany, 3-6 July 2012.

104. Nikolic, J.; Burri, M.; Rehder, J.; Leutenegger, S.; Huerzeler, C.; Siegwart, R. A UAV system for inspection of industrial facilities. In Proceedings of the IEEE Aerospace Conference, Big Sky, MT, USA, 2-9 March 2013; pp. 1-8.

105. DB about Using Drones against Graffiti Sprayers. Available online: http://www.bbc.com/ news/world-europe-22678580 (accessed on 22 December 2014).

106. SNCF Using Drones for Railway Viaduct Inspection. Available online: http://www.personal-drones.net/french-trains-company-sncf-is-starting-to-experiment-with-drones-for safety-and-inspection-purposes/ (accessed on 22 December 2014).

107. ELIMCO Industrial Aerial Inspection Solutions. Available online: http://www.elimco.com/eng/ 1_Infrastructure-Monitoring-and-Inspection_46.html (accessed on 22 December 2014).

108. Microdrones UAVs. Available online: http://www.microdrones.com/en/applications/ areas-of-application/inspection/ (accessed on22 December 2014).

109. Drones in railway maintenance. Available online: http://www.smartrailworld.com/how_drones_ are_already-being-used-by-railways-around-the-world (accessed on 22 December 2014).

110. OpenCV Feature Detectors. Available online: http://docs.opencv.org/modules/features2d/doc/ common_interfaces_of_feature_detectors.html (accessed on 30 March 2015).

111. Páll, E. Vision-Based Quadcopter Navigation for Following Indoor Corridors and Outdoor Railways. Master's Thesis, The Technical University of Cluj-Napoca, Cluj-Napoca, Romania, June 2014.

112. AR.Drone Autonomy GPS Waypoints Package. Available online: https://github.com/ AutonomyLab/ardrone_autonomy/tree/gps-waypoint (accessed on 8 May 2015).

113. Parrot AR.Drone Flight Recorder. Available online: http://ardrone2.parrot.com/ apps/flight-recorder/ (accessed on 8 May 2015).

(c) 2015 by the authors; licensee MDPI, Basel, Switzerland. This article is an open access article distributed under the terms and conditions of the Creative Commons Attribution license (http://creativecommons.org/licenses/by/4.0/). 